\title{
ARTICLE
}

Received 18 Apr 2013 | Accepted 21 Aug 2013 | Published 18 Sep 2013 DOl: 10.1038/ncomms3490

\section{Val66Met polymorphism of BDNF alters prodomain structure to induce neuronal growth cone retraction}

\author{
Agustin Anastasia ${ }^{1}$ Katrin Deinhardt ${ }^{2,3}$, Moses V. Chao ${ }^{2}$, Nathan E. Will ${ }^{1}$ Krithi Irmady ${ }^{1}$, Francis S. Lee ${ }^{4}$, \\ Barbara L. Hempstead ${ }^{1} \&$ Clay Bracken ${ }^{5}$
}

A common single-nucleotide polymorphism (SNP) in the human brain-derived neurotrophic factor (BDNF) gene results in a Val66Met substitution in the BDNF prodomain region. This SNP is associated with alterations in memory and with enhanced risk to develop depression and anxiety disorders in humans. Here we show that the isolated BDNF prodomain is detected in the hippocampus and that it can be secreted from neurons in an activitydependent manner. Using nuclear magnetic resonance spectroscopy and circular dichroism, we find that the prodomain is intrinsically disordered, and the Val66Met substitution induces structural changes. Surprisingly, application of Met66 (but not Val66) BDNF prodomain induces acute growth cone retraction and a decrease in Rac activity in hippocampal neurons. Expression of p75 NTR and differential engagement of the Met66 prodomain to the SorCS2 receptor are required for this effect. These results identify the Met66 prodomain as a new active ligand, which modulates neuronal morphology.

\footnotetext{
${ }^{1}$ Department of Medicine, Weill Cornell Medical College of Cornell University, 1300 York Avenue, New York, New York 10065, USA. ${ }^{2}$ Skirball Institute, New York University School of Medicine, 540 First Avenue, New York, New York 10016, USA. ${ }^{3}$ Centre for Biological Sciences and Institute for Life Sciences, University of Southampton, Life Sciences Building 85, Southampton, SO17 1BJ, UK. ${ }^{4}$ Department of Psychiatry, Weill Cornell Medical College of Cornell University, 1300 York Avenue, New York, New York 10065, USA. ${ }^{5}$ Department of Biochemistry, Weill Cornell Medical College of Cornell University, 1300 York Avenue, New York, New York 10065, USA. Correspondence and requests for materials should be addressed to B.L.H. (email: blhempst@med.cornell.edu) or to C.B. (email: wcb2001@med.cornell.edu).
} 
common single-nucleotide polymorphism (SNP) in the human brain-derived neurotrophic factor $(B D N F)$ gene is highly associated with abnormalities in episodic memory, a reduction in the hippocampal volume and enhanced risk for depression and anxiety disorders in humans ${ }^{1-8}$. This SNP (rs6265) is observed in more than $25 \%$ of the human population (Database of Single-Nucleotide Polymorphisms-National Center for Biotechnology Information, National Library of Medicine, 2012), and it leads to a nucleotide change from a guanine to an adenine at position $196(\mathrm{G} 196 \mathrm{~A})$ that results in a valine (Val) to methionine (Met) substitution at codon 66 (Val66Met).

$\mathrm{BDNF}$ is translated as a precursor protein (proBDNF) consisting of an amino-terminal prodomain and a carboxyterminal mature domain (mBDNF). The Val66Met substitution site is within the prodomain (Supplementary Fig. S1). ProBDNF can be cleaved by furin or proconvertases in the trans-Golgi network or secretory vesicles, respectively ${ }^{9}$. Both mBDNF and proBDNF can be secreted from neurons ${ }^{10,11}$ in an activitydependent manner after $\mathrm{KCl}$-induced depolarization ${ }^{10}$ or electrical stimulation ${ }^{11}$. In addition, proBDNF can be cleaved extracellularly by plasmin generated by tissue plasminogen activator or by selective matrix metalloproteinases including MMP3, MMP7 (ref. 12) and MMP9 (ref. 13) to release mBDNF. The fate of the isolated BDNF prodomain after proBDNF intracellular or extracellular proteolysis is not known. mBDNF binds the tropomyosin-related kinase $\mathrm{B}$ receptor to promote neuronal survival, differentiation, neurogenesis and synaptic plasticity. Modest changes in mBDNF levels in rodents result in alterations in the hippocampal function and behavioural abnormalities. In contrast, uncleaved proBDNF promotes apoptosis by binding to a receptor complex of $\mathrm{p} 75$ neurotrophin receptor $\left(\mathrm{p} 75^{\mathrm{NTR}}\right)$ and sortilin (a Vps10p-domain sorting receptor family member) ${ }^{14}$. Interestingly, recent reports have shown that proBDNF ${ }^{15}$ as well as unprocessed pro-nerve growth factor (proNGF) ${ }^{16}$ induce growth cone retraction in cultured neurons.

The BDNF prodomain is highly conserved with a valine at or near position 66 in more than 70 species examined to date (Supplementary Fig. S1). The prodomain mediates an interaction with sortilin ${ }^{17}$, which acts as a chaperone to direct intracellular trafficking of proBDNF to the regulated secretory pathway. The Met66 proBDNF variant exhibits decreased binding to sortilin, altered intracellular trafficking and a reduction in the activitydependent secretion of $\mathrm{mBDNF}^{2,6,17,18}$. Moreover, a knock-in mouse that expresses Met66 BDNF recapitulates many specific phenotypic properties of the human polymorphism ${ }^{6}$. Thus, the proposed mechanism by which Met66 BDNF mediates central nervous system effects is indirect, by a reduction in activitydependent release of mBDNF leading to altered synaptic plasticity. However, the high-sequence conservation of the BDNF prodomain (Supplementary Fig. S1), and the recent evolutionarily emergence of the Val66Met polymorphism (only present in humans) prompted us to ask whether the isolated prodomain might function as an independent ligand. We have found that the prodomain is present at high levels in vivo and is secreted from neurons in an activity-dependent manner. We further considered whether the Val66 and Met66 prodomains exhibit different structures to elicit distinct biological activities.

Here we show that structural changes induced by Val66Met substitution confer bioactivity to the prodomain, as only the Met66 prodomain acutely alters neuronal morphology. Finally, we demonstrate that the prodomain exerts this effect through differential interaction with SorCS2, the sortilin-related Vps10pdomain sorting receptor 2. Our findings suggest that the Met66 prodomain activity is an additional mechanism that may contribute to the increased incidence of depression and anxiety disorders found in humans with the SNP.

\section{Results}

BDNF prodomain is expressed in the hippocampus and secreted. The hippocampus is a key regulator of learning and memory and dysfunction of its neural circuitry underlies the development of memory impairment, depression and anxiety disorders ${ }^{19}$. To determine if the BDNF prodomain may affect the structure of hippocampal neurons, we first examined whether the prodomain was present in this brain region. Detection of the isolated prodomain in vitro and in vivo has been technically challenging due to low antibody sensitivity. However, glutaraldehyde fixation of proteins to the transfer membranes following sodium dodecyl sulphate polyacrylamide gel electrophoresis (SDSPAGE), and the use of a BDNF prodomain-specific monoclonal antibody previously characterized ${ }^{10}$, facilitated its detection in the mouse hippocampus as a $15.5 \mathrm{kDa}$ band (Fig. 1a). In order to demonstrate specificity of detection, we observed that the prodomain levels are reduced by half in $B d n f$ heterozygous $\left(B d n f^{+/-}\right)$ mouse hippocampal lysates compared with wild-type (WT) littermates (Fig. 1b). As the prodomain contains an $N$-linked glycosylation site (Supplementary Fig. S1), we performed enzymatic removal of the $N$-linked glycosylation, which resulted in migration of the prodomain at $12.3 \mathrm{kDa}$, consistent with the predicted molecular weight by its amino-acid sequence (Fig. 1c).

During late embryonic and early postnatal murine development, the expression of the prodomain in the hippocampus of C57BL/6 mice was negligible (Fig. 1d,e). However, the prodomain was detected at postnatal day 5, its expression increased significantly at 1 month and plateaued in adult mice (3-9 months) (Fig. 1d,e). The prodomain was detectable in the hippocampi of both WT Bdnf $f^{\mathrm{Val} / \mathrm{Val}}$ and $B d n f^{\text {Met/Met }}$ knock-in animals ${ }^{6}$. However, the levels of expression significantly differ, as $B d n f^{M e t / M e t}$ mice displayed lower levels of expression as compared with $B d n f^{V a l} \mathrm{Val}$ in all the time points studied (Fig. 1f,g). Other commercially available antibodies to the prodomain of BDNF (ANT-006, Alomone, Jerusalem, Israel) failed to detect the endogenous prodomain in isolation and showed very low sensitivity in detecting the recombinant prodomains (Supplementary Fig. S2).

To determine if the prodomain was secreted, we cultured the rat hippocampal neurons in conditions to reduce glia contamination and collected the conditioned media of day 6 in vitro (DIV6) cultures. The endogenous prodomain was detected in the media, and prodomain levels were increased significantly after depolarization with $25 \mathrm{mM} \mathrm{KCl}$ for $6 \mathrm{~h}$ (Fig. 2a,b). Lactate dehydrogenase (LDH) assays demonstrated there was no significant release by the cells under these culture conditions after depolarization with $\mathrm{KCl}$ (arbitrary $\mathrm{LDH}$ release units: control $0.21 \pm 0.01 ; \mathrm{KCl} 0.20 \pm 0.01)$. Incubation of the hippocampal neuron cultures from DIV3 to DIV6 with $\alpha$-2-antiplasmin and/or MMP inhibitor II (which inhibits MMP1, 3, 7 and 9) to prevent extracellular cleavage of secreted proBDNF did not significantly alter the levels of the prodomain secreted in the media in basal conditions or after depolarization (Fig. 2a,b). Enzymatic removal of $N$-linked glycosylation resulted in a reduction of the molecular mass of the endogenous secreted prodomain from $15.5 \mathrm{kDa}$ to the predicted $12.3 \mathrm{kDa}$ (Fig. 2c) as described above for the prodomain expressed in the hippocampus. We next compared the activity-dependent secretion of the prodomain from hippocampal-cortical cultures obtained from $B d n f^{V a l / V a l}$ and $B d n f^{M e t / M e t}$ knock-in mice ${ }^{6}$. We found that both Val66 and Met66 prodomains can be secreted after depolarization with $\mathrm{KCl}$; however, the levels of secreted Met66 prodomain were significantly lower as compared with the Val66 prodomain (Fig. 2d,e). These results suggest that the prodomain is detectable in the adolescent and adult murine hippocampus and that neurons secrete it. 


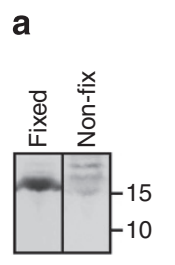

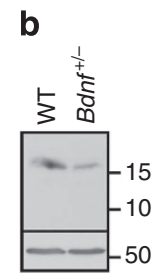

C

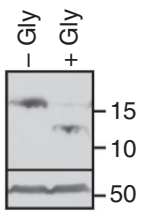

f

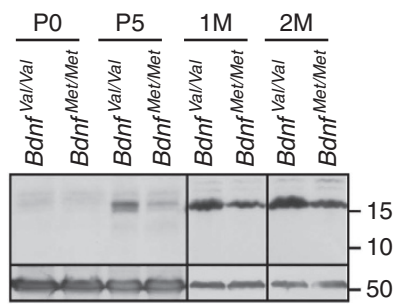

Top IB: Prodomain mAb287 Bottom IB: tubulin

d

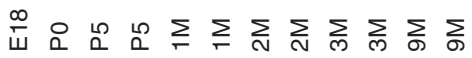
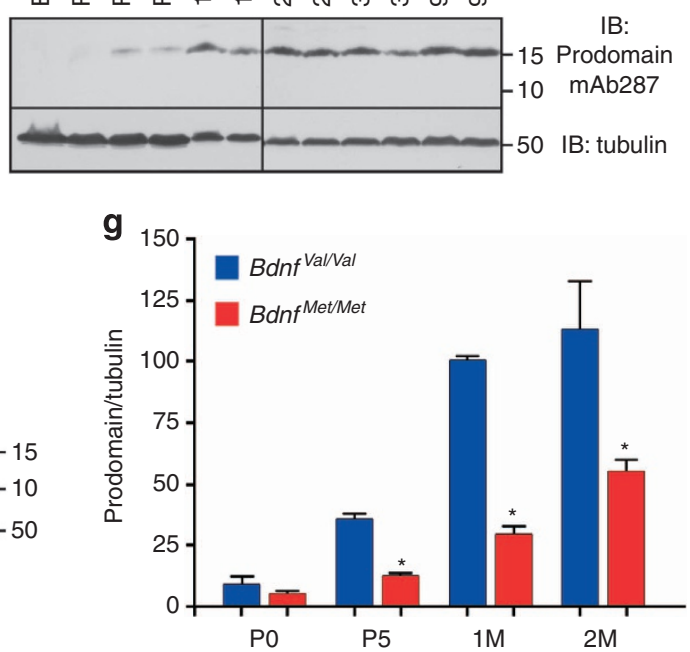

e

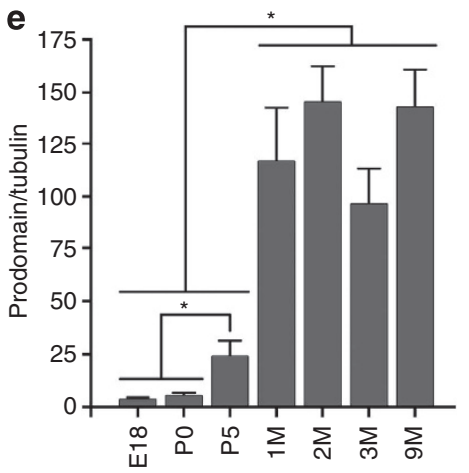

Figure 1 | The BDNF prodomain is detected at high levels in vivo. (a) Detection of BDNF prodomain (15.5kDa.) from mice hippocampus after

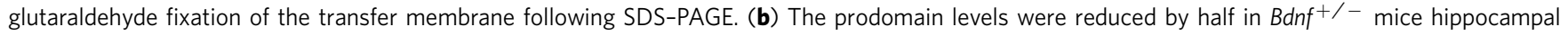
lysates, compared with WT. (c) Treatment of the hippocampal lysates with $N$-glycanase ( + Gly) reduced the molecular weight of the prodomain to

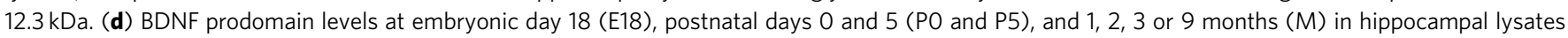
of C57BL/6 mice. (e) Quantification of (d). Bars represent mean \pm s.e.m. of prodomain densitometry data normalized to $\beta$ tubulin; $n=3$ per

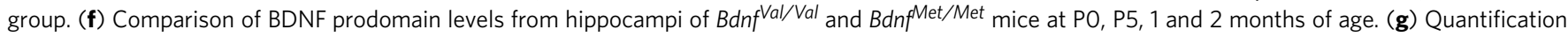
of (f). Bars represent mean \pm s.e.m. of prodomain densitometry data normalized to $\beta$ tubulin; $n=4$ per group. Statistical comparisons were made by one-way analysis of variance test. ${ }^{\star} P<0.05$.
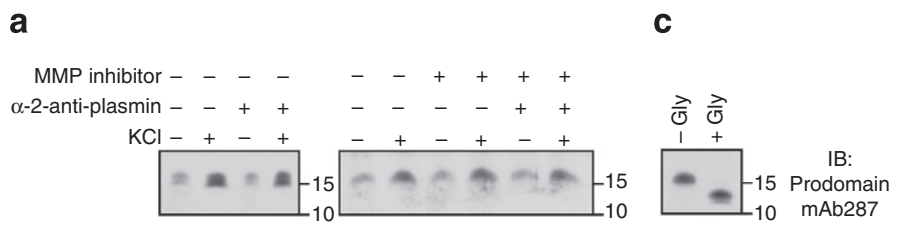

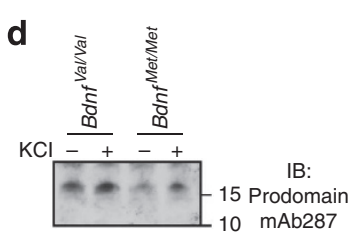

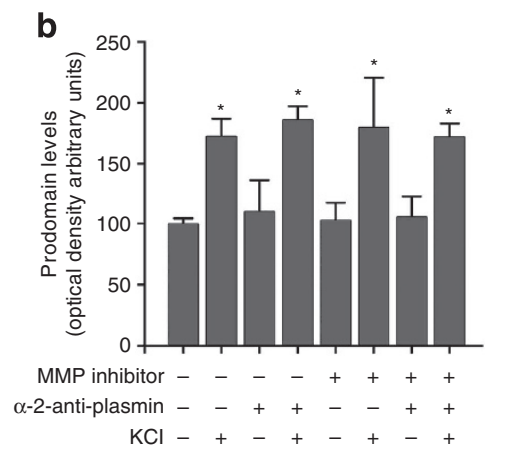

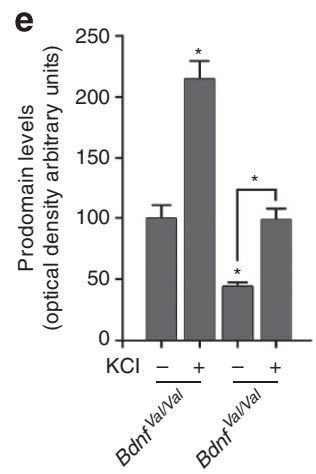

Figure 2 | The BDNF prodomain is secreted from cultured neurons. (a) Prodomain was detected in the media of DIV6 rat hippocampal cultures. Cultures were treated with or without $\alpha-2$ anti-plasmin and/or MMP inhibitor II (MMP1, 3, 7 and 9 inhibitor) in order to prevent extracellular cleavage of secreted proBDNF. Activity-dependent secretion was achieved by depolarization with $\mathrm{KCl}$. (b) Quantification of (a). Bars represent mean \pm s.e.m. of prodomain densitometry data; $n=3$ per group. (c) Treatment with $N$-glycanase reduced the molecular mass of the secreted prodomain. (d) Prodomain was detected in the media of DIV6 hippocampal-cortical neurons from E18 pups obtained from BdnfMet/ + x Bdnf Met/ + mice litters. Level of secreted prodomain differ between Bdnf $f^{V a l / V a l}$ and Bdnf Met/Met cultures. (e) Quantification of (d). Bars represent mean \pm s.e.m. of prodomain densitometry data; $n=4$ per group. Statistical comparisons were made by one-way analysis of variance test. ${ }^{\star} P<0.05$.

Val66Met substitution alters the BDNF prodomain structure. To evaluate if there are structural differences in the Val66 and Met66 prodomains, we produced both human recombinant prodomains in Escherichia coli (residues 23-113 displayed in red in Supplementary Fig. S1). The purity of the recombinant prodomains was examined by Coomassie blue and silver staining (Supplementary Fig. S3a,b). By SDS-PAGE, the recombinant prodomains migrate at the predicted molecular weight of 
a

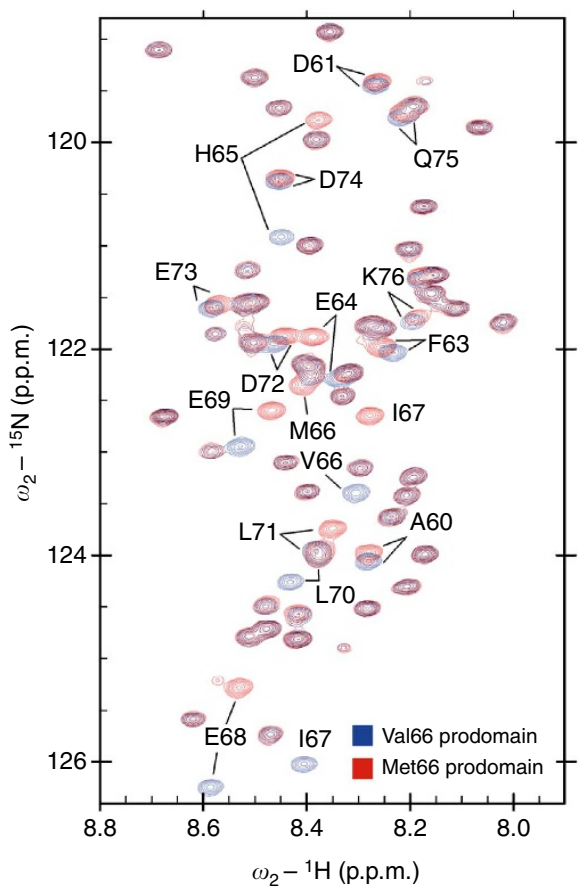

b

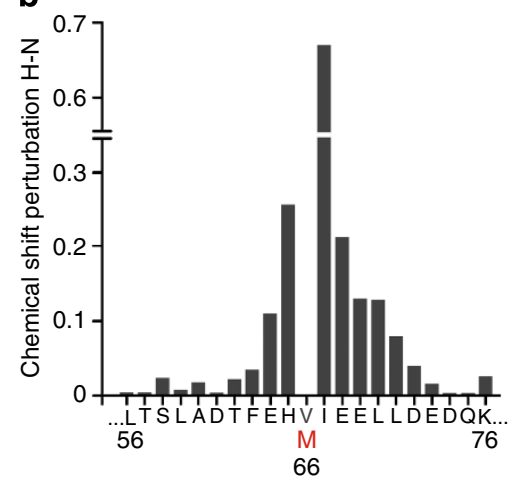

Figure 3 | Impact of the Val66Met substitution on the structure of the BDNF prodomain. (a) Overlay of the heteronuclear single-quantum coherence (HSQC) spectrum on the Val66 (blue) and Met66 (red) prodomains. Each cross-peak (chemical shift) corresponds to one residue within the sequence of the prodomain (one chemical shift for each covalently bonded pair of ${ }^{1} \mathrm{H}-{ }^{15} \mathrm{~N}$ atoms assigned to specific amides within the prodomain sequence). Full HSQC spectra of Val66 and Met66 prodomains are available in Supplementary Fig. S5. The backbone chemical shifts for the prodomains are deposited in the Biological Magnetic Resonance Bank: Val66 ID number: 19358. Met66 ID number: 19357. (b) Chemical shift deviation $(\Delta \delta)$ between BDNF Val66 and Met66 prodomains showed that changes induced by the substitution are localized to seven residues (E64, H65, 167, E68, E69, L70, L71) neighbouring the Val66Met substitution site. The variation in $\Delta \delta$ for residues 23-55, and 77-113 outside the display window was between 0.007 and 0.0037 p.p.m.

$10.2 \mathrm{kDa}$, with no difference in the detection of the Val66 and Met66 prodomains by the specific prodomain antibody used (Supplementary Fig. S3c). The BDNF prodomain is predicted to be disordered by structure prediction software (Supplementary Fig. S4). This predicted disorder in the BDNF prodomain is consistent with the absence of a defined trace of the related nerve growth factor prodomain in the proNGF-p $75^{\mathrm{NTR}}$ crystal lattice, indicating a lack of ordered structure ${ }^{20}$.

To define the structural effects of the Val66Met polymorphism, we examined the Met66 and Val66 prodomains using nuclear magnetic resonance (NMR). The ${ }^{1} \mathrm{H}-{ }^{15} \mathrm{~N}$ heteronuclear correlation spectra indicated that the Val66 and Met66 prodomains are intrinsically disordered, lacking stable secondary or tertiary structure (Fig. 3, Supplementary Fig. S5). Secondary chemical shifts analysis for the Val66 prodomain and the Met66 prodomain indicate that both are primarily disordered structures (Supplementary Fig. S6). Moreover, in the NMR conditions employed, both prodomains were monomeric showing no evidence of oligomerization or concentration-dependent change in spectral properties (from 2 to $450 \mu \mathrm{M}$ ). To define precise Val66Met structural differences, we generated sequence specific ${ }^{1} \mathrm{H} /{ }^{13} \mathrm{C} /{ }^{15} \mathrm{~N}$ heteronuclear $\mathrm{NMR}$ assignments for the Val66 and Met66 prodomains using established triple-resonance NMR methods ${ }^{21}$. This technique assigns individual resonance frequencies to specific atoms within each amino acid in the prodomain sequence (chemical shifts displayed as peaks in Fig. 3a and Supplementary Fig. S5a,b). The NMR chemical shifts provide a sensitive indicator of conformational preference within disordered states. Comparison of chemical shift deviations between the Val66 prodomain and the Met66 prodomain indicated that there are significant changes localized to seven residues (E64, H65, I67, E68, E69, L70, L71) neighbouring the Val66Met substitution site (Fig. 3a,b). The backbone chemical shifts for the prodomains are deposited in the Biological Magnetic Resonance Bank: Val66 ID number: 19358; Met66 ID number: 19357.

Val66 and Met66 prodomains transient secondary structure. We estimated the prodomain secondary structure tendency based on the heteronuclear chemical shifts using the secondary structure propensity (SSP) score $^{22}$. The positive SSP scores estimate regions of helical preference, whereas the negative scores indicate the residues with $\beta$-structure propensity. Upon Val66Met substitution, a shift in conformation from $\beta$-strand to helical conformation was observed around the position 66 (Fig. 4a). Outside the substitution area, the Val66 and Met66 prodomain SSP scores were coincident with regions of transient helix centred at residues $24,44,57$ and 90 , and $\beta$-structure that appears neighbouring residues 32, 50, 80, 101 and 110 (Fig. 4a). Chemical shift analysis using Talos + secondary structure analysis software ${ }^{23}$ showed a strong preference for $\beta$-strand around position 66 in the Val66 prodomain. The Met66 prodomain has reduced tendency to adopt $\beta$-strand as compared with the Val66 prodomain (Fig. 4b,c) consistent with the SSP score analysis.

In order to corroborate the SSP analysis and Talos + results with an NMR-independent technique, we performed circular dichroism (CD) spectroscopy. The negative peak around $200 \mathrm{~nm}$ for both prodomains $\mathrm{CD}$ spectra revealed the predominant natively unfolded conformation, whereas the changes in CD intensity at $222 \mathrm{~nm}$ are characteristic of helix propensity (Fig. 4d) 

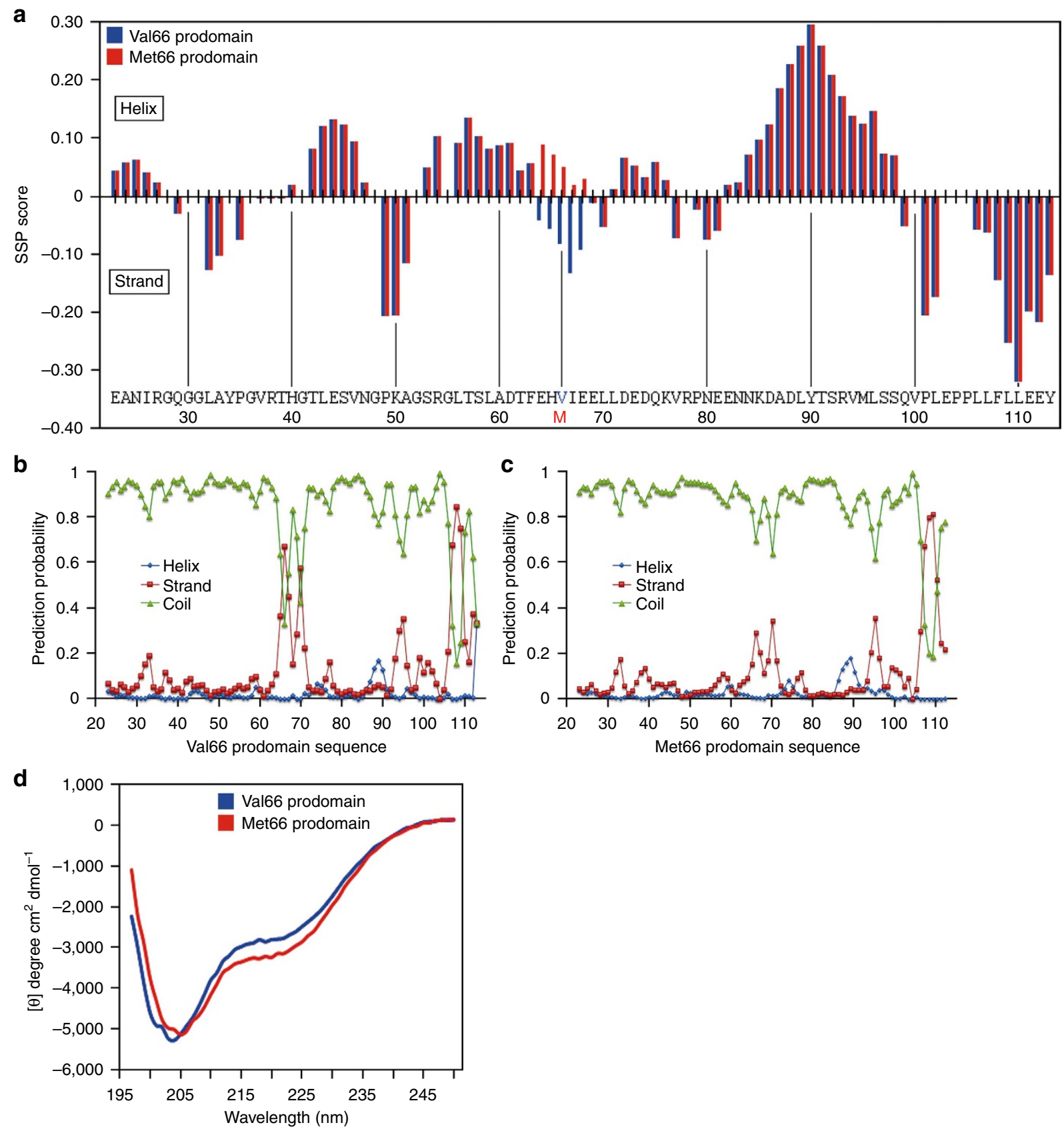

Figure 4 | Val66 and Met66 prodomains differ in transient secondary structure. (a) SSP score obtained using the backbone ${ }^{1} \mathrm{H},{ }^{15} \mathrm{~N},{ }^{13} \mathrm{C}$ chemical shifts from the BDNF prodomain Val66 (blue bars) and Met66 (red bars). The SSP score identified regions of transient structure formation (positive values $=\alpha$-helix, negative values $=\beta$-sheet). (b,c) Graphs illustrating secondary structure prediction by TALOS + analysis using the heteronuclear backbone chemical shifts of Val66 (b) and Met66 (c) prodomains. $\alpha$-helix in blue spheres, $\beta$-strand in red squares and disorder in green triangles. TALOS + analysis showed decreased $\beta$-sheet propensity in the Met66 prodomain compared with the Val66 prodomain, result that is consistent with the SSP score. (d) Ultraviolet circular dichroism (CD) spectra of $30 \mu \mathrm{M}$ of the Val66 (blue) and Met66 (red) prodomains collected in $10 \mathrm{mM} \mathrm{NaH}_{2} \mathrm{PO}_{4}$ and $50 \mathrm{mM} \mathrm{NaCl}$ $\mathrm{pH} 7.0$ at $23^{\circ} \mathrm{C}$. The negative peak around $200 \mathrm{~nm}$ revealed the natively unfolded conformation of both prodomains. However, the lower absorption at $222 \mathrm{~nm}$ for Met66 prodomain is consistent with increased tendency to helical conformation compared with the Val66 prodomain. Each spectrum is representative of four averaged scans and is normalized to the spectrum of buffer alone.

Met66 prodomain CD spectra showed a more negative absorption at $222 \mathrm{~nm}$ consistent with an increased helical tendency as compared with the Val66 prodomain (Fig. 4d), a result that is in agreement with the SSP and Talos + data. To summarize, both NMR and CD analysis demonstrate that the Val66 prodomain has increased tendency to $\beta$-structure in the residues adjacent to the position 66, whereas the Met66 prodomain displays increased helical propensity.
BDNF Met66 prodomain induces growth cone retraction. The formation of neuronal networks depends upon the growth and retraction of nascent neuronal processes. Alterations in synaptic network organization influence the occurrence and progression of neuropsychiatric disorders ${ }^{19,24}$. Therefore, we assessed the bioactivity of Val66 and Met66 prodomains in mediating acute morphological changes in the hippocampal neurons using a growth cone retraction assay ${ }^{16}$. Unprocessed proNGF has been 
a

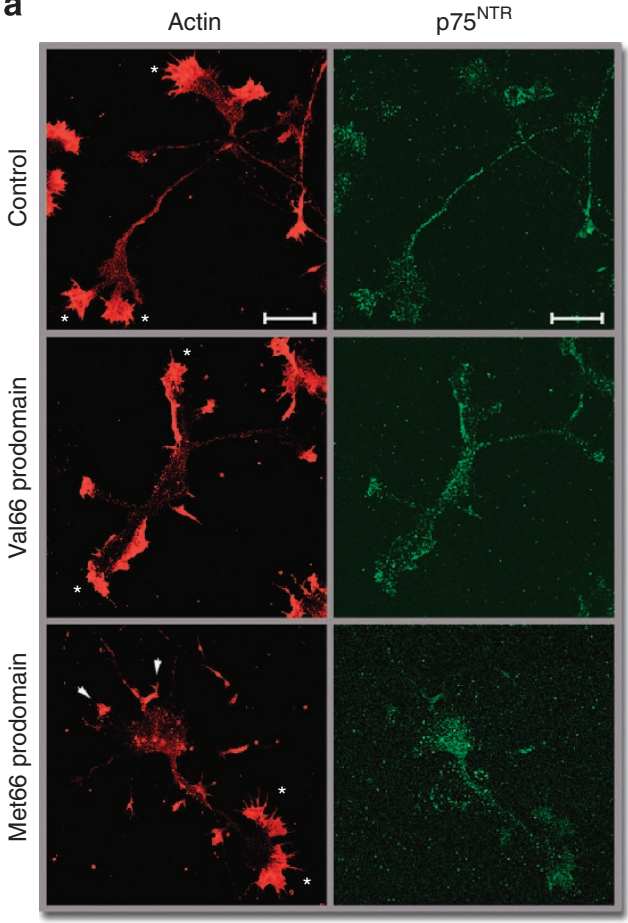

b

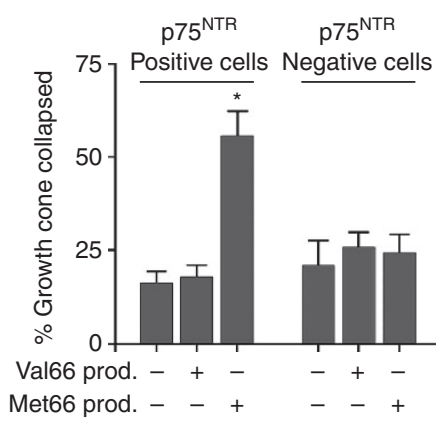

Figure 5 | The Met66 prodomain induces growth cone retraction in cultured hippocampal neurons. (a) Neurons were treated with Val66 or Met66 prodomains $\left(10 \mathrm{ng} \mathrm{ml}^{-1}\right.$ ) for $20 \mathrm{~min}$, fixed and stained for actin and $\mathrm{p} 75^{\mathrm{NTR}}$. Arrows indicate retracted growth cones; asterisks indicate intact growth cones. Scale bar, $20 \mu \mathrm{m}$. (b) Quantification of growth cone retraction in p75 NTR -positive cells shown in (a), compared with p75 NTR -negative cells. Prodomain abbreviated as prod. (b) $n=4$ independent experiments. Bars represent mean \pm s.e.m. Statistical comparisons were made by one-way analysis of variance test. ${ }^{\star} P<0.05$.

recently identified as a ligand that initiates acute retraction of growth cones, an effect mediated by the expression of $\mathrm{p} 75^{\mathrm{NTR}}$ and SorCS2, a member of the sortilin family of receptors ${ }^{16}$. A recent study indicates that proBDNF also induces growth cone retraction $^{15}$, an effect that we have confirmed (Supplementary Fig. S7a). Thus, we examined whether BDNF prodomain in isolation could induce the same effect. We treated primary mouse hippocampal neurons with recombinant Val66 or Met66 prodomains. Surprisingly, the Met66 prodomain-induced rapid growth cone retraction over a period of $20 \mathrm{~min}$, while the Val66 prodomain was inactive (Fig. 5a,b). This effect was achieved with $1 \mathrm{nM}$ $\left(10 \mathrm{ng} \mathrm{ml}^{-1}\right)$ concentration of the Met66 prodomain. At $50 \mathrm{ng} \mathrm{ml}^{-1}$, the Val66 prodomain also remained inactive without showing significant growth cone retraction (Supplementary Fig. S8). As with proNGF ${ }^{16}$ and proBDNF${ }^{15}$ (Supplementary Fig. S7a), the growth cone retraction effect induced by the Met66 prodomain was limited to $\mathrm{p} 75^{\mathrm{NTR}}$-positive cells within the culture (Fig. 5b).

Met66 prodomain binds differentially to SorCS2. Prior experiments have established that $\mathrm{p} 75^{\mathrm{NTR}}$ and sortilin interact to form a receptor complex on the cell surface ${ }^{25}$, and that SorCS2 facilitates the interaction of $\mathrm{p} 75^{\mathrm{NTR}}$ with downstream signalling proteins to promote growth cone retraction ${ }^{16}$. We were unable to detect sortilin in these culture conditions, but SorCS2 was expressed in $\mathrm{p} 75^{\mathrm{NTR}}$-positive cells ${ }^{16}$. Thus, we tested whether the prodomain interacts with SorCS2 and p $75^{\mathrm{NTR}}$ using co-immunoprecipitation analysis of cells expressing the prodomain with either of the receptors. We were unable to detect interaction of the prodomains with $\mathrm{p} 75^{\mathrm{NTR}}$ (Fig. 6a). However, both Met66 and Val66 prodomains interact with SorCS2
(Fig. 6b). By quantitative analysis, the Met66 prodomain interacted more effectively with SorCS2 than the Val66 prodomain (Fig. 6b,c). Moreover, we performed NMR spectroscopy of the Val66 prodomain or the Met66 prodomains with human recombinant SorCS2 to determine if the prodomains interacted differently with this receptor. Consistent with the co-immunoprecipitation result, both Val66 and Met66 prodomains interacted with SorCS2 as detected by chemical shift perturbation in the prodomains upon addition of the receptor (Fig. 6d, Supplementary Fig. S9a,b). Amino acids 34, $38-40,51,53-54,76-77,80,96$ and 100-111 of both Val66 and Met66 prodomains interact with SorCS2 as shown by chemical shift deviation upon addition of the receptor (Fig. 6d). Surprisingly, the Met66 prodomain displayed greater chemical shift changes upon interaction to SorCS2 between residues 65 and 71, whereas the same residues in the Val66 prodomain do not engage the receptor (Fig. 6d, under \#). This represents a novel region of interaction in the prodomain that is conferred by the Met66 substitution. These larger chemical shift changes in the Met66 prodomain upon SorCS2 binding may reflect a tighter interaction or a different binding conformation. Thus, although both prodomains interact with SorCS2, the interactions are distinct, and this difference can result in the differential activity of the prodomains.

To further confirm association of the Val66 or Met66 prodomains with SorCS2, we performed diffusion NMR measurements. Measurements of translational diffusion are highly sensitive to changes in hydrodynamic shape, which can be influenced by changes in intramolecular conformation and intermolecular associations. Association of the prodomains $(10.2 \mathrm{kDa})$ with the ectodomain of SorCS2 $(113 \mathrm{kDa})$ will influence the prodomain solution mobility and the apparent radius of hydration. The measured diffusion rates of Val66 and Met66 prodomains 
a

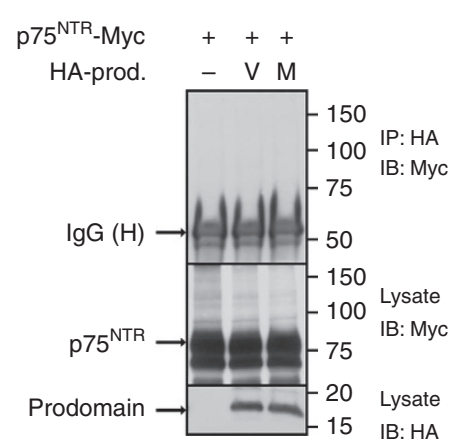

b

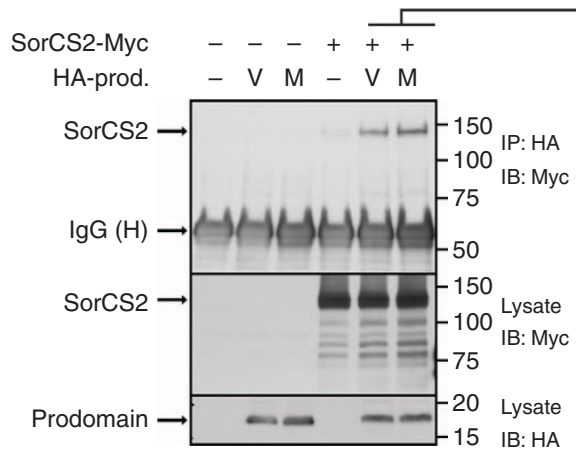

C

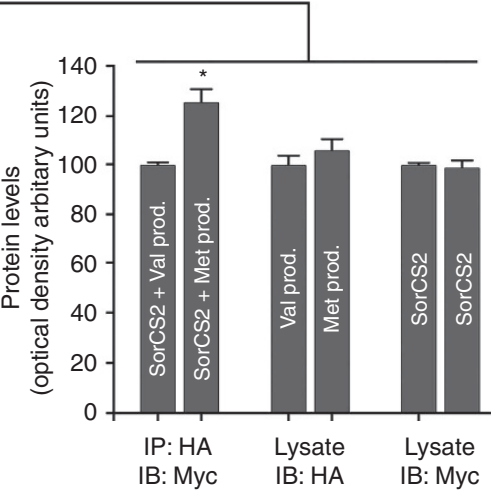

d

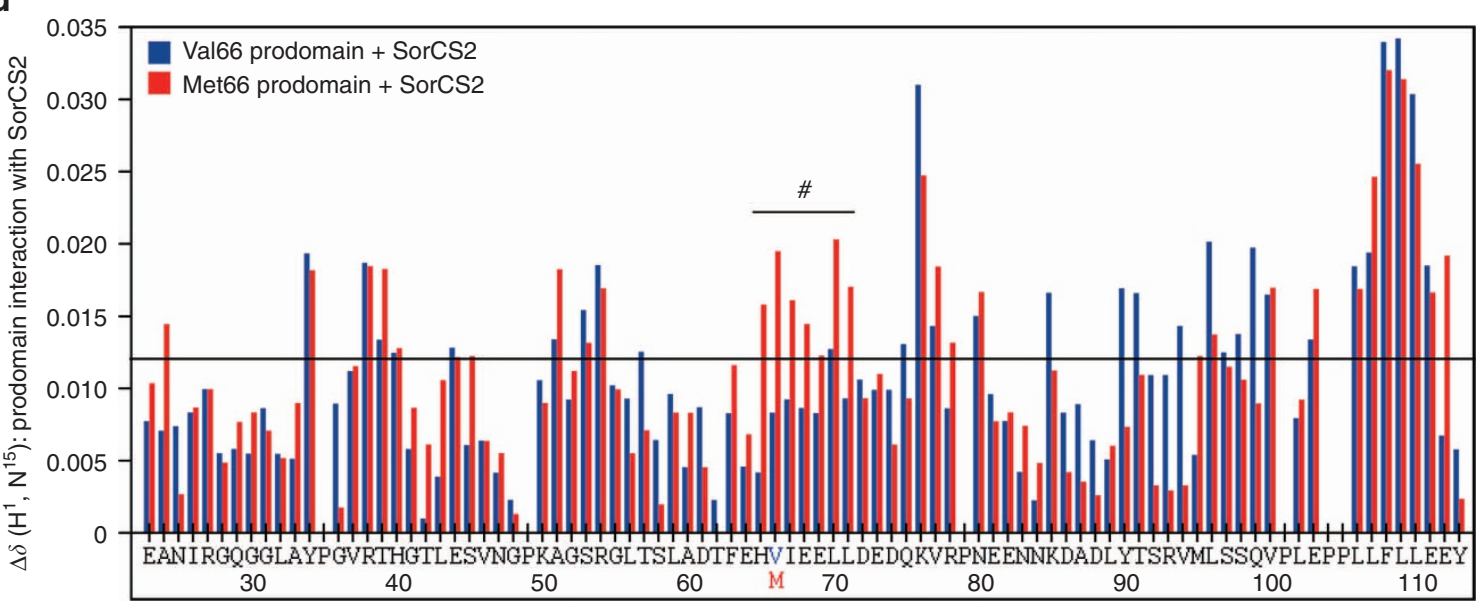

e

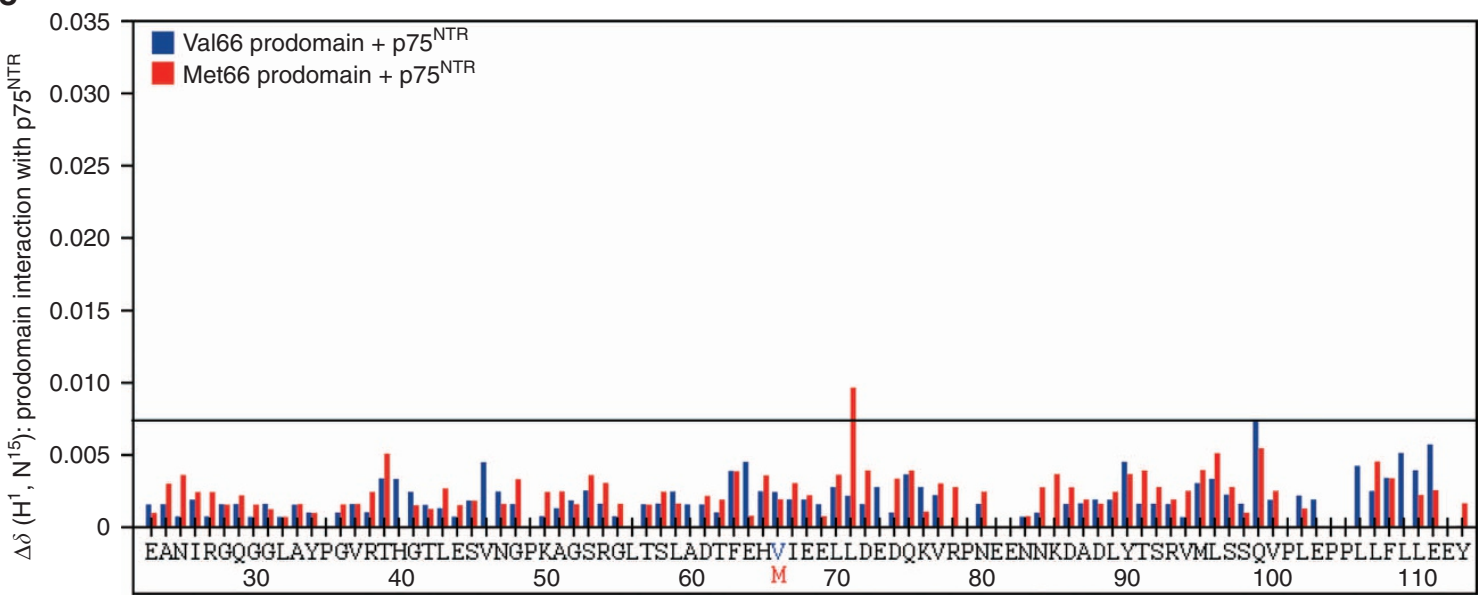

Figure 6 | The Val66 and Met66 prodomains binds differently to SorCS2 but do not bind to p75 $\mathbf{N T R}^{\mathbf{N}}$. (a,b) HEK293T cells were transfected with the indicated constructs, the lysates were immunoprecipitated with anti-HA antibody, followed by detection with indicated antibodies. $\mathrm{V}=\mathrm{Val} 66$ prodomain and $\mathrm{M}=$ Met66 prodomain constructs. (a) We were unable to detect interaction of either prodomains with p $75^{\mathrm{NTR}}$ by co-immunoprecipitation. Representative blot of four independent experiments. (b) Both Val66 and Met66 prodomains co-immunoprecipitated with SorCS2; however, Met66 prodomain interacted $\sim 23 \%$ more than the Val66 as quantified in (c). (c) $n=9$ independent experiments. Bars represent mean \pm s.e.m. Statistical comparisons were made by one-way analysis of variance test. ${ }^{\star} P<0.05$. Interaction of Val66 and Met66 prodomains with SorCS2 (d) or p75 NTR (e) was also assessed by NMR spectroscopy. $\Delta \delta=$ chemical shift deviation between Val66 and Met66 prodomains together with the receptors. Interaction of the prodomain with SorCS2 (d) was assessed at $4.5 \mu \mathrm{M}$ for both proteins and data were collected at $600 \mathrm{MHz}$. The Met 66 prodomain displayed greater chemical shift changes upon interaction to SorCS2 between residues 65 and 71 (shown under \#) compared with the Val66 prodomain. Interaction of the prodomain with $\mathrm{p} 75^{\mathrm{NTR}}(\mathbf{e})$ was assessed at $6.6 \mu \mathrm{M}$ for both proteins and data were collected at $800 \mathrm{MHz}$. Interaction of the prodomain with p75 $\mathrm{NTR}$ was undetectable by NMR. Standard deviation (s.d.) was calculated for all the analysis and a threshold line equivalent to 2 s.d. was drawn to show the limit of what is considered interaction. 
were $1.02 \times 10^{-10} \mathrm{~m}^{2} \mathrm{~s}^{-1}$ and $1.04 \times 10^{-10} \mathrm{~m}^{2} \mathrm{~s}^{-1}$, respectively. Upon addition of SorCS2, Val66 display a slight decrease in diffusion $D_{\mathrm{T}}^{\text {Val66 }}=0.99 \times 10^{-10} \mathrm{~m}^{2} \mathrm{~s}^{-1}$, whereas the Met66 displayed significant reduction in diffusion rates to $D_{\mathrm{T}}^{\text {Met66 }}=0.74$ $\times 10^{-10} \mathrm{~m}^{2} \mathrm{~s}^{-1}$, corresponding to more than a doubling of the apparent hydrodynamic volume (Supplementary Table S1). This indicates that the Met66 prodomain interacts with SorCS2 in a greater temporal extent as compared with the Val66 prodomain, and confirms that the Val66 and Met66 prodomains interact differentially with SorCS2.

We could not detect significant structural changes in SorCS2 after either prodomain binding using intrinsic tryptophan fluorescence spectroscopy. Using tryptophan excitation at $285 \mathrm{~nm}$, emission spectra with maxima at $329 \mathrm{~nm}$ was collected with SorCS2 alone as a control. The addition of Val66 or Met66 prodomain to SorCS2 showed emission maxima at $329 \mathrm{~nm}$ of 98.1 and $97.1 \%$ of the control, respectively. This is consistent with the lack of structural changes in Sortilin after neurotensin binding ${ }^{26}$. Significant chemical shift changes of either prodomain were not observed after the addition of p75 ${ }^{\mathrm{NTR}}$ by NMR (Fig. 6e, Supplementary Fig. S9c,d) confirming the lack of binding found by co-immunoprecipitation analysis. In contrast to the isolated prodomains, proBDNF was able to interact with SorCS2 and $\mathrm{p} 75^{\mathrm{NTR}}$ assessed by co-immunoprecipitation analysis (Supplementary Fig. S7b).

SorCS2 is required for Met66-induced growth cone retraction. As the prodomains interact with SorCS2, we evaluated whether SorCS2 is involved in Met66 prodomain-induced retraction of growth cones. To address this, we pre-incubated the hippocampal cultures with function-blocking antibodies directed against the SorCS2 ectodomain before the addition of the ligands. Addition of antibodies did not affect neuronal morphology, but blocked the ability of the Met66 prodomain to induce growth cone retraction (Fig. 7a). Control immunoglobulin Gs did not impair the effect (Fig. 7a). Moreover, anti-SorCS2 antibodies also blocked the ability of proBDNF to induce growth cone retraction (Supplementary Fig. S7a). To further confirm the involvement of SorCS2 in growth cone retraction triggered by the Met66 prodomain, we infected the hippocampal cultures with lentivirus expressing small hairpin RNA (shRNA) targeting SorCS2. SorCS2 shRNA expression resulted in a partial but significant decrease in SorCS2 protein levels as compared with the scrambled shRNA infection (Fig. 7b). The infection with scrambled shRNA resulted in higher growth cone retraction in basal conditions without ligand addition (Fig. 7c). However, the shRNA-induced SorCS2 decrease resulted in reduced growth cone retraction after Met66 prodomain administration, a result that is in agreement with the effects of function-blocking SorCS2 antibody experiments.

Growth cones are rich in actin filament-containing structures such as lamellipodia and filopodia. The dynamic extension and retraction of these actin structures are regulated by Rho GTPases family members including $\mathrm{Rac}^{27}$. We previously showed that proNGF induces displacement of the guanine nucleotide exchange factor Trio from $\mathrm{p} 75^{\mathrm{NTR}}$ and SorCS2, thereby decreasing local activity of Rac to induce growth cone retraction ${ }^{16}$. To determine whether the Val66 prodomain, the Met66 prodomain or proBDNF lead to differential signalling downstream of SorCS2, we isolated and quantified activated Rac a

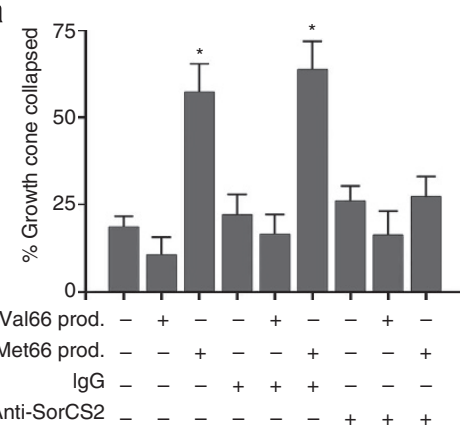

d

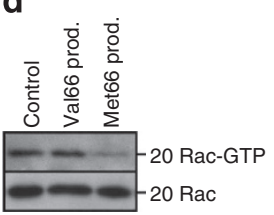

b

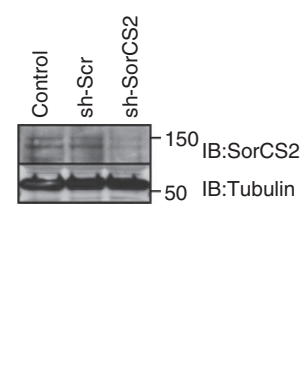

C

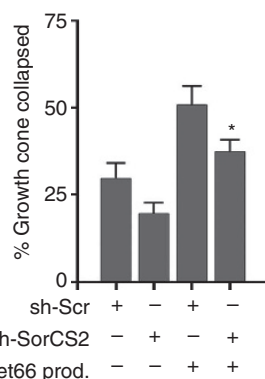

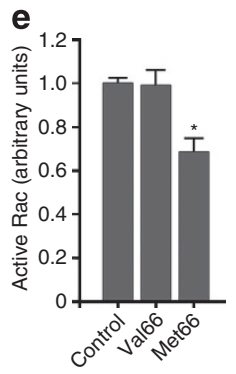

Figure 7 | Met66-SorCS2 interaction is required to induce growth cone retraction. (a) SorCS2 antibodies were able to block the Met66 prodomaininduced growth cone retraction. Neurons were pre-incubated with anti-SorCS2 (or control immunoglobulin Gs), treated with Val66 or Met66 prodomains $\left(10 \mathrm{ng} \mathrm{ml}^{-1}\right)$ for $20 \mathrm{~min}$, and followed by growth cone retraction analysis. Quantification assessed in p75 ${ }^{\mathrm{NTR}}$ positive cells from three independent experiments. (b) Representative blot showing SorCS2 expression in cultured hippocampal neurons after knock-down using SorCS2 shRNA (sh-SorCS2) lentivirus infection, as compared with uninfected controls or scrambled shRNA (sh-Scr)-infected cells. (c) SorCS2 partial downregulation achieved with SorCS2 shRNA was able to partially prevent the Met66 prodomain-induced growth cone retraction. Quantification of four independent experiments. (d) Only Met66 prodomain administration, but not the Val66, induced a decrease in Rac activity in cultured hippocampal neurons. The hippocampal neurons were incubated with Val66 or Met66 prodomain for 20 min, and cell lysates were incubated with GST-PAK-CRIB beads to isolate activated Rac (Rac-GTP). (e) Quantification of (d). Activated Rac was measured by densitometry and normalized to total Rac in the input. Quantification assessed in four independent experiments. Bars represent mean \pm s.e.m. Statistical comparisons were made by one-way analysis of variance test. ${ }^{\star} P<0.05$. 
using the Cdc42/Rac interactive binding (CRIB) domain of the Rac effector p21-activated kinase (PAK-CRIB). Surprisingly, the Met66 prodomain or proBDNF exposure led to a significant decrease in Rac activity in the cultured hippocampal neurons, as compared with the absence of effect in Val66 prodomain-treated or in untreated neurons (Fig. 7d,e; Supplementary Fig. S7c).

The results strongly suggest that the helical preference in the Met66 prodomain is advantageous in interacting with SorCS2 and inducing active binding that is reflected in downregulation of Rac activity and growth cone retraction. In contrast, the Val66 prodomain with $\beta$-structure tendency remains inactive.

\section{Discussion}

We found that the variant Met66 prodomain acutely alters neuronal morphology, as it induces growth cone retraction. Mechanistically, we showed that the Val66Met substitution induces a shift in transient secondary structure from $\beta$-sheet to a helical conformation in the residues surrounding the substitution. This shift confers bioactivity to the Met66 prodomain through differential interaction with SorCS2, the sortilin-related Vps10p-domain sorting receptor 2. Moreover, we have demonstrated that the BDNF prodomain is detectable at high levels in vivo and that it is secreted in an activity-dependent manner from the cultured hippocampal neurons.

In previous studies, the isolated BDNF prodomain was undetectable ${ }^{10}$. It is known that low-molecular-weight proteins can be detected more efficiently by cross-linking the peptides to the transfer membrane using glutaraldehyde ${ }^{28,29}$. Glutaraldehyde fixation of the proteins to the transfer membrane following SDS-PAGE and the use of a prodomain-specific monoclonal antibody previously characterized ${ }^{10}$, allow reliable detection of the prodomain in the murine central nervous system. However, we cannot rule out the possibility that the fixative promotes an antigen retrieval effect unmasking the prodomain epitopes. We corroborate that both the intracellular and the secreted prodomains are $\mathrm{N}$-linked glycosylated as predicted by the amino-acid sequence. Furthermore, the developmental production of the prodomain parallels the expression of $\operatorname{mBDNF}^{10,30,31}$.

The hippocampal expression of the prodomain in the $B d n f^{M e t / M e t}$ mice is less than the observed in control Bdnf $\mathrm{Val} / \mathrm{Val}$ mice, which is in agreement with a prior study that reported diminished hippocampal BDNF expression for the Bdnf Met/Met mice by enzyme-linked immunosorbent assay ${ }^{32}$. The Val66Met polymorphism leads to a decrease in the trafficking of BDNF to secretory vesicles and the subsequent impairment of activitydependent release of $\mathrm{BDNF}^{2,17,18}$. The decreased levels of prodomain could in part be due to decreased transport and/or secretion from afferent inputs to the hippocampus such as the cortex or sub-cortical areas. Alternatively, it was reported previously that the Met66 BDNF transcripts show decreased dendritic trafficking ${ }^{33}$, and this may result in decreased local translation and secretion in the hippocampus. We cannot exclude the possibility that the Met66 proBDNF precursor exhibits decreased cleavage to its mature counterpart and prodomain, or that there is preferential degradation of the Met66 prodomain. Nonetheless, we were able to detect the Met66 prodomain in the $B d n f^{M e t / M e t}$ mice hippocampus indicating that it may exert local bioactivity.

Both $\mathrm{mBDNF}^{34}$ and proBDNF${ }^{10,11}$ are secreted in an activitydependent manner. Similarly, here we demonstrate that the release of the prodomain from neurons is also regulated by activity. Inhibition of extracellular cleavage of secreted proBDNF using $\alpha-2$ anti-plasmin and an inhibitor of numerous matrix metalloproteinases did not significantly change the levels of the secreted prodomain in our culture conditions, suggesting that most of the cleavage of proBDNF to prodomain and mBDNF may occur in intracellular compartments. It has been reported that there is impaired regulated secretion of mBDNF from $B d n f^{M e t / M e t}$ neuronal cultures ${ }^{6}$. In agreement with these studies, we found that there is a significant decrease in the activitydependent release of the endogenous Met66 prodomain as compared with the Val66 prodomain. However, regardless of the secreted levels, both are detected in the media suggesting that they may act as ligands for paracrine or autocrine signalling.

The Val66 and Met66 prodomains were determined to be intrinsically disordered with transient structural features using both NMR and CD spectroscopy. We detected specific conformational changes in the residues neighbouring the Val66Met substitution area, from $\beta$-structure propensity in the Val66 prodomain to helical conformation tendency in the Met66. It is known that small changes in local structure can substantially alter protein activity. This is particularly true in the case of intrinsically disordered proteins, which have prominent roles in signal transduction, antigen-antibody recognition, intracellular trafficking and as hormones, as well as in a number of neurodegenerative diseases such as Alzheimer's, Huntington's, Parkinson's, prion and frontotemporal dementia diseases ${ }^{35,36}$. For instance, structural changes of similar magnitude to the ones observed for Val66Met substitution are observed in the A53T substitution in $\alpha$-synuclein, which has been correlated to impact the kinetics of aggregation of this protein and toxicity in Parkinson's disease ${ }^{37}$. The structural changes between Val66 and Met66 prodomain impact on their interaction with SorCS2 receptor, as the Met66 with more helical propensity, shows greater local chemical shifts perturbation during binding. We document that both Val66 and Met66 prodomains interact with SorCS2; however, they engage differentially as observed by NMR (Fig. 6d). Residues 65-71 of the Met66 prodomain interact with SorCS2, whereas the same residues in the Val66 prodomain do not engage the receptor, and this represents a novel region of interaction in the prodomain that is conferred by the Met66 substitution. This differential binding can explain the different bioactivity between the prodomains; however, other differences such as differential aggregation, bio-availability of the prodomains, differential trafficking or interaction of the prodomains with unknown factors may also have a role. This differential binding was confirmed by NMR diffusion measurements and co-immunoprecipitation experiments, and resulted in Rac inactivation only after Met66 addition to the cultured neurons.

The development, maturation and plasticity of neuronal networks rely on the emergence, path finding and retraction of neuronal processes. Alterations in synaptic network organization influence the occurrence and progression of neuropsychiatric disorders ${ }^{19,24,38}$. ProNGF has been recently identified to activate p $75^{\text {NTR }}$ and SorCS2 to promote fascin phosphorylation and its dissociation from actin filaments resulting in neurite retraction ${ }^{16}$. ProBDNF is also able to induce growth cone retraction as shown in Supplementary Fig. S7a confirming previously published studies ${ }^{15}$. Here we describe that the Met66 prodomain is sufficient to initiate growth cone retraction. As shown by NMR, the Met66 prodomain engages differently with SorCS2, but not with $p 75^{\mathrm{NTR}}$. However, Met66 prodomain is only active in p75 ${ }^{\mathrm{NTR}}$-expressing cells suggesting that $\mathrm{p} 75^{\mathrm{NTR}}$ acts in a complex with SorCS2 or is downstream of SorCS2.

ProBDNF interacts with both SorCS2 and $\mathrm{p} 75^{\mathrm{NTR}}$ and induces growth cone retraction. This may contribute to the bioactivity of proBDNF in this assay, whereas the Val66 prodomain that binds in a disadvantageous manner to SorCS2 is inactive. In early postnatal ages both proBDNF and processed products (mBDNF and prodomain) are present in the hippocampus. During adulthood, mBDNF and the prodomain are the most abundant 
forms, whereas proBDNF levels are decreased ${ }^{10}$. From this, we can infer that the actions of proBDNF may predominate in early postnatal time points, but the actions of the prodomain may prevail during adulthood.

Most growth factors are synthesized as precursors, and prodomains are thought to function primarily to promote protein folding, intracellular sorting or to shield the mature domain from receptors to limit bioavailability ${ }^{39}$. Here we have demonstrated that the endogenous BDNF prodomain is detected in the hippocampus, and is secreted in an activity-dependent manner from neurons. Most importantly, the evolutionarily recent SNP that results in the Val66Met substitution in the prodomain promotes growth cone retraction in hippocampal neurons with involvement of $\mathrm{p} 75^{\mathrm{NTR}}$ and binding to SorCS2, in contrast to inactive Val66 prodomain. A function of the Val66 prodomain remains to be elucidated and future studies with mature neuronal networks are required to understand other prodomain actions.

The endophenotypes related to neuropsychiatric disorders associated with the Val66Met polymorphism have been linked with decreased sorting and secretion of BDNF, thus impairing neuronal differentiation and plasticity via reduced tropomyosinrelated kinase $\mathrm{B}$ receptor activation ${ }^{32,33}$. Here we show that the Met66 prodomain is a newly identified ligand that selectively activates SorCS2 to acutely alter neuronal morphology, and we propose that this effect is an additional mechanism that contributes to altered neural plasticity in humans with the SNP.

\section{Methods}

Animals. C57BL/6 male mice from different ages (as specified in the figures and captions) were maintained with a 12-h light-dark cycle and with free access to water and food. Val66Met knock-in mice $\left(B d n f^{M e t / M e t}\right)$ and WT $\left(B d n f^{V a l / V a l}\right)$ were used as reported previously ${ }^{6}$. Briefly, the BDNF coding region was replaced by a targeting vector with the BDNF sequence with or without a point mutation (G196A), which was introduced to result in the Val66Met substitution. These BDNF knock-in alleles are regulated by the endogenous BDNF promoters. Heterozygous $B d n f^{V a l / M e t}$ mice were intercrossed to yield $B d n f^{V a l / V a l}, B d n f^{V a l} / \mathrm{Met}$ and $B d n f^{\text {Met } / M e t}$ offspring at Mendelian rates. Heterozygous $B d n f^{+/}$mice $\left(B d n f^{t m 1 J a e}\right)$ were purchased from The Jackson Laboratory (Bar Harbour, ME, USA). Sprague-Dawley pregnant rats and C57BL/6 pregnant mice were purchased from Taconic Farms (Hudson, NY, USA) and Charles River (Wilmington, MA, USA), respectively. Animal care was in accordance with Weill Medical College of Cornell University IACUC.

SDS-PAGE and western blot. Dissected hippocampi or HEK293T (ATCC, Manassas, VA, USA) cells were lysed in 1\% triton X-100 (Sigma, St Louis, MO, USA), 1\% nonidet P-40 (Roche, Indianapolis, IN, USA), 10\% glycerol, in buffer Tris-buffered saline pH 7.4 supplemented with protease inhibitor cocktail (Sigma). Protein concentration was determined by Bradford (Bio-Rad, Hercules, CA, USA). For deglycosylation, $100 \mu \mathrm{g}$ of lysates or $100 \mu \mathrm{l}$ of conditioned media were incubated with $1 \mu \mathrm{l}$ of $\mathrm{N}$-glycanase (ProZyme, San Leandro, CA, USA) for $1 \mathrm{~h}$ at $37^{\circ} \mathrm{C}$. The lysates or conditioned media were run in SDS-PAGE, the proteins were transferred to a $0.45-\mu \mathrm{m}$ polyvinylidene fluoride (PVDF) membrane (Millipore, Billerica, MA, USA), and the membranes were fixed with $2.5 \%$ glutaraldehyde (Sigma) in PBS pH 7.4 unless otherwise written. After blocking with 5\% bovine serum albumin (BSA) in Tris-buffered saline with $0.1 \%$ Tween 20 , membranes were incubated with the prodomain antibody ${ }^{10}\left(\mathrm{mAb} 287,1: 2000,12-16 \mathrm{~h}\right.$ at $4{ }^{\circ} \mathrm{C}$, GeneCopoeia, Rockville, MD, USA), anti- $\beta$ tubulin (Sigma, 1:15000, $1 \mathrm{~h}$ at $20-25^{\circ} \mathrm{C}$ ) or anti-SorCS2 (R\&D Systems, Minneapolis, MN, USA, 1:1000, $12-16 \mathrm{~h}$ at $4^{\circ} \mathrm{C}$ ) followed by anti-mouse horseradish peroxidase (HRP) secondary antibody (1:5,000 for prodomain, or $1: 15,000$ for $\beta$-tubulin, $1 \mathrm{~h}$ at $20-25^{\circ} \mathrm{C}$, Calbiochem-Millipore) or anti-sheep HRP (1:5,000 for SorCS2, $1 \mathrm{~h}$ at $20-25^{\circ} \mathrm{C}$, Calbiochem-Millipore). Bands were analysed by enhanced chemiluminescence (Amersham-GE, Pittsburgh, PA, USA), and the densitometry was analysed using ImageJ (1.45, NIH, Bethesda, MD, USA, http://rsbweb.nih.gov/ij/). Quantization was normalized to $\beta$-tubulin for each individual sample. Full gel scans are provided in Supplementary Fig. S10.

Hippocampal cultures for prodomain secretion analysis. Primary hippocampal neurons were isolated from E18 Sprague-Dawley rat embryos. Neurons were dissociated with $0.05 \%$ trypsin at $37^{\circ} \mathrm{C}$ for $20 \mathrm{~min}$ followed by trituration with firepolished glass pipettes. Cells plated on poly-D-lysine (Sigma)-coated dishes were grown in Neurobasal medium (Gibco, Grand Island, NY, USA) containing B27, $1 \mathrm{mM}$ pyruvate (Gibco), $2 \mathrm{mM}$ glutamine (Gibco), and $10 \mathrm{mM} 5$ - fluorodeoxyuridine (Sigma). From day 3 in vitro to day 6, $\alpha$-2-anti-plasmin (100 $\mu \mathrm{M}$, Calbiochem-Millipore) and/or MMP Inhibitor II (10 $\mu \mathrm{M}$, CalbiochemMillipore; MMP1 IC $50=24 \mathrm{nM}$, MMP3 $\mathrm{IC}_{50}=18.4 \mathrm{nM}, \mathrm{MMP} 7 \mathrm{IC}_{50}=30 \mathrm{nM}$, and MMP9 $\mathrm{IC}_{50}=2.7 \mathrm{nM}$.) were added to the cultures. DIV6 media was collected and protease inhibitor cocktail (Sigma) was added. For depolarization experiments, $25 \mathrm{mM} \mathrm{KCl}$ was added to the cultures for $6 \mathrm{~h}$. To study the secretion of the endogenous

Val66 and Met66 prodomains, we cultured hippocampal-cortical neurons from E18 pups obtained from $B d n f^{M e t /+} \times B d n f^{M e t /+}$ litters. Each pup dissected area was plated in a different well and genotyped as described ${ }^{6}$. Subsequently, depolarization and harvesting of the culture media were performed as described for rat hippocampal cultures above.

LDH release assay. Conditioned media $(50 \mu \mathrm{l})$ from DIV6 neurons in culture (with or without depolarization with $\mathrm{KCl}$ ) was harvested and analysed for LDH levels using a colorimetric assay following the manufacturer's protocol (CytoTox 96; Promega, Madison, WI, USA).

Expression and purification of recombinant BDNF prodomains. The plasmid pET28 (Novagen, Madison, WI, USA) containing the gene of human BDNF prodomain Val66 or Met66 (amino acids 23-113 preceded by an N-terminal His-tag and SUMO $)^{40}$, was transformed in BL21(DE3)pLysS competent cells (Invitrogen, Grand Island, NY, USA). Proteins were produced using the Marley media swap method for isotopic labelling $\left({ }^{15} \mathrm{~N}\right.$ and $\left.{ }^{13} \mathrm{C} /{ }^{15} \mathrm{~N}\right)$ of recombinant proteins ${ }^{41}$. Prodomain samples were purified using a nickel resin column (Ni-NTA, Invitrogen). SUMO was cleaved by Ulp-1 (His6 tagged) proteolysis. Subsequently, the sample was negatively selected in a second nickel resin column where the now untagged BDNF prodomain was obtained in the flow though fraction. The resulting protein was precipitated using acetone, resuspended and dialyzed in $50 \mathrm{mM} \mathrm{NaH} \mathrm{PO}_{4} 100 \mathrm{mM} \mathrm{NaCl} \mathrm{pH} \mathrm{7.0.} \mathrm{The} \mathrm{sample} \mathrm{purity} \mathrm{was}$ assessed by SDS-PAGE using Coomassie blue and silver staining methods (Supplementary Fig. S3). The prodomains concentrations were calculated using random-coil UV absorbance in water $\left(\varepsilon_{(280)}=4,470 \mathrm{M}^{-1} \mathrm{~cm}^{-1}\right)^{42}$, and confirmed using the Bradford method.

NMR spectroscopy. NMR samples were prepared in $93 \% \mathrm{H}_{2} \mathrm{O} / 7 \% \mathrm{D}_{2} \mathrm{O}, 50 \mathrm{mM}$ $\mathrm{NaH}_{2} \mathrm{PO}_{4} 100 \mathrm{mM} \mathrm{NaCl} \mathrm{pH} 7.0$ at concentrations varying from $2 \mu \mathrm{M}$ to $450 \mu \mathrm{M}$. NMR spectra were acquired on a Bruker Avance $500 \mathrm{MHz}$, a Varian INOVA $600 \mathrm{MHz}$ spectrometer both at Weill Cornell Medical College, and a Bruker Avance $800 \mathrm{MHz}$ spectrometer at the New York Structural Biology Center. The $600 \mathrm{MHz}$ and $800 \mathrm{MHz}$ spectrometers were equipped with triple-resonance cryogenic probes. The $500 \mathrm{MHz}$ spectrometer was equipped with a BBO room temperature probe and NMR chemical shift assignments were performed at $7^{\circ} \mathrm{C}$. NMR data were processed using the software nmrPipe ${ }^{43}$ and analysed using Sparky ${ }^{44}$. Secondary structure predictions based on the observed chemical shifts were performed using the SSP ${ }^{22}$ and TALOS $+{ }^{23}$ programs. Chemical shift deviation: $\Delta \delta=\left[\left(\Delta \delta^{1} \mathrm{H}\right)^{2}+\left(0.153^{*} \Delta \delta^{15} \mathrm{~N}\right)^{2}\right]^{1 / 2}$, where $\Delta \delta^{1} \mathrm{H}$ and $\Delta \delta^{15} \mathrm{~N}$ are the ${ }^{1} \mathrm{H}$ and ${ }^{15} \mathrm{~N}$ chemical shifts change, and 0.153 is a weighting factor for ${ }^{15} \mathrm{~N}$ shifts. Interaction of the prodomain with recombinant human SorCS2 (R\&D Systems) was assessed at $4.5 \mu \mathrm{M}$ for both proteins and data were collected at $600 \mathrm{MHz}$. Interaction of the prodomain with human recombinant $\mathrm{p} 75^{\mathrm{NTR}}$ (R\&D Systems) was assessed at $6.6 \mu \mathrm{M}$ for both proteins and data were collected at $800 \mathrm{MHz}$. Data were processed and analysed using Topspin software (Bruker Instruments version 2.1). Sequential backbone resonance assignments were achieved using standard three dimensional $\mathrm{HNCO}$, HNCA, HN(CO)CA, HNCACB and CBCA(CO)NH resonance heteronuclear experiments ${ }^{45}$

NMR diffusion measurements. Gradient diffusion measurements were acquired using the BPP-LED pulse sequence ${ }^{46}$ at $500 \mathrm{MHz}$ in a Bruker Avance spectrometer, at $25^{\circ} \mathrm{C}$. Samples were all run in a volume constricted Shigemi tube, using a 20 -mm sample height between plunger and tube bottom, to minimize convection artifacts. Data were collected at $298 \mathrm{~K}$ using 32 gradient experiments with linearly increasing $z$-gradient strengths from 2 to $95 \%$ of the maximum $55.7 \mathrm{G} \mathrm{cm}^{-1}$, while maintaining a constant $6 \mathrm{~ms}$ gradient delay over the $100 \mathrm{~ms}$ diffusion period. The hydrodynamic radius was calibrated using $10 \mathrm{mM}$ dioxane as an internal standard $^{47}$. The data were processed and analysed using Bruker topspin software version 2.1. The hydrodynamic radius is calculated using the Stokes-Einstein equation $D_{\mathrm{T}}=k_{\mathrm{B}} T\left(6 \pi \eta R_{\mathrm{h}}\right)^{-1}$ where $D_{\mathrm{T}}$ is the translational diffusion coefficient, $k_{\mathrm{B}}$ is Boltzmann constant, $T$ is temperature in Kelvin, $\eta$ is the solvent viscosity and $R_{\mathrm{h}}$ is the hydrodynamic radius, by using dioxane as an internal reference the radius can be calculated $R_{\mathrm{h}}=\left(D_{\mathrm{T}}^{\text {dioxane }} / D_{\mathrm{T}}\right) R_{\mathrm{h}}^{\text {dioxane }}$, assuming $R_{\mathrm{h}}^{\text {diovane }}=2.12 \AA$.

Intrinsic tryptophan fluorescence spectroscopy. Fluorescence data were collected on a spectrophotometer (Photon Technology International) at $20^{\circ} \mathrm{C}$ with measurements from $305 \mathrm{~nm}$ to $500 \mathrm{~nm}$ in $1 \mathrm{~nm}$ increments. SorCS2, Val66 prodomain and Met66 prodomain were used at $0.17 \mu \mathrm{M}$ in $50 \mathrm{mM}$ Tris, $100 \mathrm{mM} \mathrm{NaCl} \mathrm{pH} \mathrm{7.0.}$ 
CD spectroscopy. CD spectra were acquired on an Aviv 62DS (Aviv Associates, Lakewood, NJ, USA) CD spectropolarimeter, using a $0.1 \mathrm{~cm}$ path length cuvette with $30 \mu \mathrm{M}$ prodomains in $10 \mathrm{mM} \mathrm{NaH}_{2} \mathrm{PO}_{4} 50 \mathrm{mM} \mathrm{NaCl} \mathrm{pH}$ 7.0. The wavelength dependence of molar ellipticity, $[\theta]$, was monitored at $23^{\circ} \mathrm{C}$ as the average of four scans, using a 5-s integration time with $1.0-\mathrm{nm}$ wavelength increments. Spectra were baseline-corrected against buffer alone.

Growth cone retraction assay. Primary hippocampal neurons were isolated from E15 C57BL/6 mouse embryos. Neurons were dissociated with $0.05 \%$ trypsin (Gibco) at $37^{\circ} \mathrm{C}$ for $8 \mathrm{~min}$ followed by trituration with fire-polished glass pipettes. Cells plated on poly-D-lysine (Sigma)-coated dishes were grown in Neurobasal medium (Gibco) containing B27 (Gibco) and $0.5 \mathrm{mM}$ glutamine (Gibco). Val66 proBDNF was collected from supernatants of HEK293FT cells (ATCC) transfected with a construct encoding human proBDNF (furin cleavage site mutated: RR128AA). proBDNF concentration was estimated by western blot (using BDNF antibody from Santa Cruz) comparing with known concentrations of recombinant BDNF (PeproTech, Rocky Hill, NJ, USA). For the assay, we added 10 or $50 \mathrm{ng} \mathrm{ml}^{-1}$ of the Val66 prodomain, Met66 prodomain or proBDNF to hippocampal neurons for $20 \mathrm{~min}$ before fixation. In order to block SorCS2, we preincubated cells with anti-SorCS2 $\left(20 \mu \mathrm{g} \mathrm{ml}^{-1}, \mathrm{R} \& \mathrm{D}\right.$ Systems) or control sheep IgG for $20 \mathrm{~min}$ on ice before addition of the prodomains or proBDNF at $37^{\circ} \mathrm{C}$. DIV3 neurons were fixed with ice-cold methanol for $10 \mathrm{~min}$. Coverslips were blocked with $10 \%$ normal donkey serum, $2 \%$ bovine serum albumin and $0.25 \%$ fish skin gelatin in Tris-buffered saline for $30 \mathrm{~min}$; incubated with anti-Actin $(1: 10,000$, Sigma, clone AC-74; anti-p75 ${ }^{\mathrm{NTR}}$ 1:1,000, R\&D Systems; both for $30 \mathrm{~min}$ at $20-25^{\circ} \mathrm{C}$ ); followed by secondary antibodies (anti-goat Alexa 488, and anti-mouse Alexa 555, 1:1,000, Invitrogen) mixed with Hoechst $(1: 10,000)$ for $30 \mathrm{~min}$ at $20-25^{\circ} \mathrm{C}$; and mounted with Mowiol 488 (Calbiochem-Millipore). Cells were imaged with an LSM 510 laser-scanning confocal microscope (with a $40 \times$ Plan Neofluor, numerical aperture 1.3 DIC oil immersion objective, Carl Zeiss, Oberkochen, Germany). Images were processed with LSM 510 software (Carl Zeiss) and ImageJ.

\section{Co-immunoprecipitation experiments in HEK293T cells. HEK293T cells} (ATCC) were grown in DMEM supplemented with $10 \%$ fetal bovine serum (Gibco) and penicillin-streptomycin. Cells were transfected using Lipofectamine 2000 (Invitrogen) with Myc-tagged p $75^{\mathrm{NTR}}$ (a kind gift of Dr. Phil Barker), Myc-tagged SorCS2, HA/Flag-tagged Val66 prodomain, HA/Flag-tagged Met66 prodomain (both prodomains constructs produced by PCR using human pcDNA3.1 proBDNF as a template), and/or proBDNF constructs. Forty-eight hours after transfection, cells were lysed, and protein concentration was determined by Bradford (Bio-Rad). Lysates were pre-cleared with protein A-sepharose (Sigma), immunoprecipitated using an HA antibody (Sigma), followed by protein A-sepharose resin (Sigma). Following SDS-PAGE, the membranes were incubated with Myc antibody $\left(1: 15,000,1 \mathrm{~h}\right.$ at $20-25^{\circ} \mathrm{C}$, Bethyl) followed by anti-rabbit HRP secondary antibody $\left(1: 15,000,1 \mathrm{~h}\right.$ at $20-25^{\circ} \mathrm{C}$, Calbiochem-Millipore), or with HA.11 antibody $\left(1: 10,000,1 \mathrm{~h}\right.$ at $20-25^{\circ} \mathrm{C}$, Covance) followed by anti-mouse HRP secondary antibody $\left(1: 10,000,1 \mathrm{~h}\right.$ at $20-25^{\circ} \mathrm{C}$, Calbiochem-Millipore).

Lentiviral vectors and transduction. Lentiviral plasmids (pLKO.1) containing shRNA against mouse SorCS2 (targeting sequence: $5^{\prime}$-CGCTGAACTCTCATAGA ATCA- $3^{\prime}$ ) and a scramble control were obtained from Open Biosystems Thermo Scientific (Waltham, MA, USA). Lentivirus was packaged by co-transfection of shRNA constructs with packaging plasmids pMD2.G and pCMV-dR8.9 using TransIT-LT transfection reagent (Mirus, Madison, WI, USA) into HEK293FT cells. Media was changed $24 \mathrm{~h}$ later, and the media supernatant was collected $48 \mathrm{~h}$ after transfection. Supernatants were filtered through a $0.45-\mu \mathrm{m}$ filter and pelleted by centrifugation with PEG-it (System Biosciences, Mountain View, CA, USA). Pellets were resuspended in Neurobasal medium (Gibco). Mouse primary hippocampal neurons were infected on DIV0 and medium was changed the following day.

Rac activity assay. Rac activity assays were performed as described previously ${ }^{48}$. Briefly, DIV2 hippocampal neurons were stimulated with Val66 prodomain, Met66 prodomains or proBDNF $\left(10 \mathrm{ng} \mathrm{ml}^{-1}\right)$ for $20 \mathrm{~min}$, and cells were lysed in lysis buffer supplemented with $10 \mathrm{mM} \mathrm{MgCl}$. Cleared lysates were incubated with glutathione S-transferase (GST) or GST-PAK-CRIB beads ( $20 \mu \mathrm{g}$ per reaction) for $30 \mathrm{~min}$ at $4{ }^{\circ} \mathrm{C}$. Isolated active Rac was analysed by western blot using a Racl antibody (Millipore, clone 23A8), and normalized to the input. GST and GST-PAK-CRIB proteins were expressed in BL21(DE3)pLysS cells (Invitrogen) followed by incubation with glutathione sepharose. The purity of the recombinant proteins was analysed by Coomassie blue staining.

\section{References}

1. Hajek, T., Kopecek, M. \& Hoschl, C. Reduced hippocampal volumes in healthy carriers of brain-derived neurotrophic factor Val66Met polymorphism: meta-analysis. World J. Biol. Psychiatry 13, 178-187 (2012).

2. Egan, M. F. et al. The BDNF val66met polymorphism affects activitydependent secretion of BDNF and human memory and hippocampal function. Cell 112, 257-269 (2003).
3. Frielingsdorf, H. et al. Variant brain-derived neurotrophic factor Val66Met endophenotypes: implications for posttraumatic stress disorder. Ann. N.Y. Acad. Sci. 1208, 150-157 (2010).

4. Soliman, F. et al. A genetic variant BDNF polymorphism alters extinction learning in both mouse and human. Science 327, 863-866 (2010).

5. Verhagen, M. et al. Meta-analysis of the BDNF Val66Met polymorphism in major depressive disorder: effects of gender and ethnicity. Mol. Psychiatry 15, 260-271 (2010).

6. Chen, Z. Y. et al. Genetic variant BDNF (Val66Met) polymorphism alters anxiety-related behavior. Science 314, 140-143 (2006).

7. Dincheva, I., Glatt, C. E. \& Lee, F. S. Impact of the BDNF Val66Met Polymorphism on Cognition: Implications for Behavioral Genetics. Neuroscientist 18, 439-451 (2012).

8. Martinowich, K., Manji, H. \& Lu, B. New insights into BDNF function in depression and anxiety. Nat. Neurosci. 10, 1089-1093 (2007).

9. Teng, K. K., Felice, S., Kim, T. \& Hempstead, B. L. Understanding proneurotrophin actions: recent advances and challenges. Dev. Neurobiol. 70, 350-359 (2010).

10. Yang, J. et al. Neuronal release of proBDNF. Nat. Neurosci. 12, 113-115 (2009).

11. Nagappan, G. et al. Control of extracellular cleavage of ProBDNF by high frequency neuronal activity. Proc. Natl Acad Sci. USA 106, 1267-1272 (2009).

12. Lee, R., Kermani, P., Teng, K. K. \& Hempstead, B. L. Regulation of cell survival by secreted proneurotrophins. Science 294, 1945-1948 (2001).

13. Mizoguchi, H. et al. Matrix metalloproteinase-9 contributes to kindled seizure development in pentylenetetrazole-treated mice by converting pro-BDNF to mature BDNF in the hippocampus. J. Neurosci. 31, 12963-12971 (2011).

14. Teng, H. K. et al. ProBDNF induces neuronal apoptosis via activation of a receptor complex of p75NTR and sortilin. J. Neurosci. 25, 5455-5463 (2005).

15. Sun, Y. et al. ProBDNF collapses neurite outgrowth of primary neurons by activating RhoA. PLoS One 7, e35883 (2012).

16. Deinhardt, K. et al. Neuronal growth cone retraction relies on proneurotrophin receptor signaling through Rac. Sci. Signal. 4, ra82 (2011).

17. Chen, Z. Y. et al. Sortilin controls intracellular sorting of brain-derived neurotrophic factor to the regulated secretory pathway. J. Neurosci. 25, 6156-6166 (2005).

18. Chen, Z. Y. et al. Variant brain-derived neurotrophic factor (BDNF) (Met66) alters the intracellular trafficking and activity-dependent secretion of wild-type BDNF in neurosecretory cells and cortical neurons. J. Neurosci. 24, 4401-4411 (2004).

19. Price, J. L. \& Drevets, W. C. Neurocircuitry of mood disorders. Neuropsychopharmacology. 35, 192-216 (2010).

20. Feng, D. et al. Molecular and structural insight into proNGF engagement of p75NTR and sortilin. J. Mol. Biol. 396, 967-984 (2010).

21. Sattlera, M., Schleucherb, J. \& Griesinger, C. Heteronuclear multidimensional NMR experiments for the structure determination of proteins in solution employing pulsed field gradients. Prog. Nucl. Magn. Reson. Spectrosc. 34, 93-158 (1999).

22. Marsh, J. A., Singh, V. K., Jia, Z. \& Forman-Kay, J. D. Sensitivity of secondary structure propensities to sequence differences between alpha- and gammasynuclein: implications for fibrillation. Protein Sci. 15, 2795-2804 (2006).

23. Shen, Y., Delaglio, F., Cornilescu, G. \& Bax, A. TALOS +: a hybrid method for predicting protein backbone torsion angles from NMR chemical shifts. J. Biomol. NMR 44, 213-223 (2009).

24. Bassett, D. S. \& Bullmore, E. T. Human brain networks in health and disease Curr. Opin. Neurol. 22, 340-347 (2009).

25. Nykjaer, A. et al. Sortilin is essential for proNGF-induced neuronal cell death Nature 427, 843-848 (2004).

26. Quistgaard, E. M. et al. Ligands bind to Sortilin in the tunnel of a ten-bladed beta-propeller domain. Nat. Struct. Mol. Biol. 16, 96-98 (2009).

27. Gonzalez-Billault, C. et al. The role of small GTPases in neuronal morphogenesis and polarity. Cytoskeleton (Hoboken.) 69, 464-485 (2012).

28. Kurien, B. T. \& Scofield, R. H. A brief review of other notable protein detection methods on blots. Methods Mol. Biol. 536, 557-571 (2009).

29. Karey, K. P. \& Sirbasku, D. A. Glutaraldehyde fixation increases retention of low molecular weight proteins (growth factors) transferred to nylon membranes for western blot analysis. Anal. Biochem. 178, 255-259 (1989).

30. Baquet, Z. C., Gorski, J. A. \& Jones, K. R. Early striatal dendrite deficits followed by neuron loss with advanced age in the absence of anterograde cortical brainderived neurotrophic factor. J. Neurosci. 24, 4250-4258 (2004).

31. Kolbeck, R., Bartke, I., Eberle, W. \& Barde, Y. A. Brain-derived neurotrophic factor levels in the nervous system of wild-type and neurotrophin gene mutant mice. J. Neurochem. 72, 1930-1938 (1999).

32. Bath, K. G. et al. BDNF Val66Met impairs fluoxetine-induced enhancement of adult hippocampus plasticity. Neuropsychopharmacology 37, 1297-1304 (2012).

33. Chiaruttini, C. et al. Dendritic trafficking of BDNF mRNA is mediated by translin and blocked by the G196A (Val66Met) mutation. Proc. Natl Acad. Sci. USA 106, 16481-16486 (2009). 
34. Goodman, L. J. et al. Regulated release and polarized localization of brainderived neurotrophic factor in hippocampal neurons. Mol. Cell Neurosci. 7, 222-238 (1996).

35. Uversky, V. N., Oldfield, C. J. \& Dunker, A. K. Intrinsically disordered proteins in human diseases: introducing the D2 concept. Annu. Rev. Biophys. 37, 215-246 (2008).

36. Tompa, P. Intrinsically disordered proteins: a 10-year recap. Trends Biochem. Sci. 37, 509-516 (2012).

37. Bussell, Jr. R. \& Eliezer, D. Residual structure and dynamics in Parkinson's diseaseassociated mutants of alpha-synuclein. J. Biol. Chem. 276, 45996-46003 (2001).

38. Dwivedi, Y. Brain-Derived Neurotrophic Factor in Suicide Pathophysiology (2012). The Neurobiological Basis of Suicide. Chapter 8. CRC Press; Boca Raton (FL).

39. Shi, M. et al. Latent TGF-beta structure and activation. Nature 474, 343-349 (2011).

40. Mossessova, E. \& Lima, C. D. Ulp1-SUMO crystal structure and genetic analysis reveal conserved interactions and a regulatory element essential for cell growth in yeast. Mol. Cell 5, 865-876 (2000).

41. Marley, J., Lu, M. \& Bracken, C. A method for efficient isotopic labeling of recombinant proteins. J. Biomol. NMR 20, 71-75 (2001).

42. Gill, S. C. \& von Hippel, P. H. Calculation of protein extinction coefficients from amino acid sequence data. Anal. Biochem. 182, 319-326 (1989).

43. Delaglio, F. et al. NMRPipe: a multidimensional spectral processing system based on UNIX pipes. J. Biomol. NMR 6, 277-293 (1995).

44. Goddard, T. D. \& Kneller, D. G. SPARKY 3 (University of Californina, San Francisco, 2002).

45. Cao, W., Bracken, C., Kallenbach, N. R. \& Lu, M. Helix formation and the unfolded state of a 52-residue helical protein. Protein Sci. 13, 177-189 (2004).

46. Wu, D. H. Chen. ,A. D. \& Johnson, C. S. An improved diffusion-ordered spectroscopy experiment incorporating bipolar-gradient pulses. J. Magn. Reson. 115, 260-264 (1995).

47. Jones, J. A. Wilkins, D. K., Smith, L. J. \& Dobson, C. M. Characterization of protein unfolding by NMR diffusion measurements. J. Biomol. NMR 10, 199-203 (1997).

48. Neubrand, V. E., Thomas, C., Schmidt, S., Debant, A. \& Schiavo, G. Kidins220/ ARMS regulates Racl-dependent neurite outgrowth by direct interaction with the RhoGEF Trio. J. Cell Sci. 123, 2111-2123 (2010).

\section{Acknowledgements}

This work was supported by the NIH (NS21072 and HD23315 to M.V.C., NS052819 to F.S.L., NS030687 and NS064114 to B.L.H. and S10-RR023694-01EWOF to C.B.) and the Human Frontier Science Program to K.D. We acknowledge the kind gift of the Myc-tagged $\mathrm{p} 75^{\mathrm{NTR}}$ construct from Dr Phil Barker (McGill University, Montreal, Canada). We thank Jianmin Yang for helpful discussion and revision of the manuscript. Parts of this work was carried out at the New York Structural Biology Center (NYSBC) supported by NIH, USA, the Keck Foundation and New York State Office of Science, Technology and Academic Research. We thank the NYSBC staff for excellent assistance.

\section{Author contributions}

A.A. performed the biochemical analysis of brain lysates, primary cultured cells and transfected heterologous cells. A.A and N.E.W. generated the constructs. A.A. and C.B. synthesized and purified the recombinant prodomains, performed circular dichroism, fluorescence and NMR spectroscopy. K.D. performed the growth cone retraction assay. K.I. generated the SorCS2 shRNA lentivirus. A.A, K.D., M.V.C., F.S.L., B.L.H. and C.B. designed the experiments and wrote the manuscript.

\section{Additional information}

Supplementary Information accompanies this paper at http://www.nature.com/ naturecommunications

Competing financial interests: The authors declare no competing financial interests.

Reprints and permission information is available online at http://npg.nature.com/ reprintsandpermissions/

How to cite this article: Anastasia, A. et al. Val66Met polymorphism of BDNF alters prodomain structure to induce neuronal growth cone retraction. Nat. Commun. 4:2490 doi: 10.1038/ncomms3490 (2013). 


\title{
Corrigendum: Val66Met polymorphism of BDNF alters prodomain structure to induce neuronal growth cone retraction
}

\author{
Agustin Anastasia, Katrin Deinhardt, Moses V. Chao, Nathan E. Will, Krithi Irmady, Francis S. Lee, \\ Barbara L. Hempstead \& Clay Bracken
}

Nature Communications 4:2490 doi: 10.1038/ncomms3490 (2013); Published 18 Sep 2013; Updated 8 Apr 2014

The glutaraldehyde fixation method used in this Article was previously published by Dieni et al. to detect BDNF propeptide, and should have been cited in the first paragraph of the Results section as follows: 'However, glutaraldehyde fixation of proteins to the transfer membranes following sodium dodecyl sulphate polyacrylamide gel electrophoresis (SDS-PAGE) according to Dieni et al., and the use of a BDNF prodomain-specific monoclonal antibody previously characterized ${ }^{10}$, facilitated its detection in the mouse hippocampus as a $15.5-\mathrm{kDa}$ band (Fig. 1a), in agreement with the findings of Dieni et al.'.

Dieni, S. et al. BDNF and its pro-peptide are stored in presynaptic dense core vesicles in brain neurons. J. Cell. Biol. 196, 775-788 (2012). 